\title{
A utilização da artéria torácica interna aumenta a mortalidade hospitalar do paciente coronariano idoso revascularizado?
}

José Carlos R. IGLÉZIAS*, Luís Alberto DALLAN*, Domingos D. LOURENÇO FILHO*, Hélio Antônio FABRI*, Antônio F. RAMIRES*, Protásio Lemos da LUZ*, Sérgio Almeida de OLIVEIRA*, Fúlvio PILEGG**, Adib D. JATENE*

RBCCV 44205-141

IGLÉZIAS, J. C. R.; DALLAN, L. A. O.; LOURENÇO FILHO, D. D.; FABRI, H. A.; RAMIRES, J. F.; LUZ, P. L.; OLIVEIRA, S. A.; PILEGGI, F.; JATENE, A. D. - A utilização da artéria torácica interna aumenta a mortalidade hospitalar do paciente coronariano idoso revascularizado? Rev. Bras. Cir. Cardiovasc., 6(2): 124-127, 1991.

RESUMO: No InCor, foi elaborado um protocolo para avaliar se o emprego da artéria torácica intema alterava a mortalidade hospitalar nos pacientes coronarianos idosos (idade $\geqslant 70$ anos), submetidos a revascularizaçāo isolada, eletiva do miocárdio. No protocolo foram incluidos parâmetros clínicos, hemodinâmicos, radiológicos, operatórios e de pós-operatório. Foram analisados 100 pacientes consecutivos, com observaçóes completas, em estudo prospectivo. A análise estatística foi realizada pelo teste de $\mathrm{X}^{2}$ de Pearson. Concluímos que houve diferença estatística entre os grupos comparados e que a mortalidade hospitalar foi maior no grupo de pacientes nos quais se utilizou o enxerto de veia safena autógena. Atribuiu-se ao cirurgião, como triador, a obtenção de tais resultados, utilizando a artéria torácica interna naqueles pacientes em melhor estado geral.

DESCRITORES: miocárdio, revasclarização, cirurgia.

\section{INTRODUÇĀO}

Um número crescente de pacientes idosos (idade $\geqslant 70$ anos) vem sendo submetido a revascularizaçāo do miocárdio, no InCor e em outros grandes centros, na última década.

Anteriormente, pacientes com idade superior a 65 anos eram recusados para este tipo de operação, porém, sabe-se hoje que, isoladamente, a idade não representa uma contra-indicação para a revascularização do miocárdio (ENNABLI \& PELLETIEN ${ }^{3}$ ).

Em análise discriminatória aplicada a 7658 pacientes submetidos a revascularização isolada do miocárdio, cita-se a idade $\geqslant 65$ anos como fator de risco para maior mortalidade operatória neste tipo de operação (GERSH et alii ${ }^{4}$ ).

No pós-operatório de pacientes idosos revascularizados, observa-se maior incidência de complicaçōes: neurológicas, pulmonares, renais e infecciosas, fato este que aumenta a permancência hospitalar, porém não contra-indica a operação (MONTAGNE et alii ${ }^{6}$ ).

Em estudo envolvendo 1995 pacientes, entre $1980-1982$, submetidos a revascularização isolada do miocárdio, COSGROVE et alii $^{2}$ concluem que a operação realizada com a utilização da artéria "mamária" é

Trabalho realizado no Instituto do Coração do Hospital das Clínicas da Faculdade de Medicina da Universidade de São Paulo. São Paulo, SP. Brasil.

Recebido para publicação em 1: de março de 1991

- Do Instituto do Coraçāo do Hospital das Clinicas da Faculdade de Medicina da Universidade de São Paulo.

Endereço para separatas: José Carlos Iglézias. Av. Dr. Enéas de Carvalho Aguiar, 44. Divisão Cirúrgica. 05403 São Paulo, SP, Brasil. 
IGLÉZIAS, J. C. R.; DALLAN, L. A. O.; LOURENÇO FILHO, D. D.; FABRI, H. A.; RAMIRES, J. F.; LUZ, P. L.; OLIVEIRA, S. A.; PILEGGI, F.; JATENE, A. D. - A utilização da artéria torácica interna aumenta a mortalidade hospitalar do paciente coronariano idoso revascularizado? Rev. Bras. Cir. Cardiovasc., 6(2): 124-127, 1991.

segura e não está associada ao aumento da morbidade, ou da mortalidade.

Comparando 532 pacientes revascularizados utilizando artérias torácicas internas com 216 pacientes revascularizados somente com veia safena, em seguimento de 15 anos, CAMERON et alii ${ }^{1}$ concluem que a artéria torácica interna permite melhor proteção contra as manifestaçōes da doença arterial coronariana do que o enxerto de veia safena autógena.

GIBSON \& LOOP ${ }^{5}$ observaram que, na idade extrema, ou em pacientes de alto risco portadores de doença pulmonar significante, deve-se evitar a abertura das pleuras e está contra-indicado o uso da artéria mamária para a revascularizaçāo do miocárdio.

Sabendo-se que a perviabilidade da artéria "mamária" é maior que a do enxerto de safena, segundo CAMERON et alii ' , que a utilização da mesma parece estar relacionada a maior morbidade, permanência hospitalar e mortalidade, desenvolvemos um projeto específico para estudar este assunto entre nós, qual seja, se a utilização da(s) artéria(s) torácica(s) interna(s) na revascularização do miocárdio a que se submete o paciente coronariano idoso (idade $\geqslant 70$ anos) acarreta maior morbidade, ou mortalidade hospitalar.

TABELA 1

\section{PARAMETROS INCLUÍDOS NA ANALISE ESTATISTICA}

1. Idade

2. Sexo

3. Diabetes Mellitus

4. Acidente Vascular Cerebral Prévio

5. Doença pulmonar obstrutiva

6. Hipertensão arterial sistêmica

7. Tabagismo

8. Angina instável

9. Obesidade $\mathrm{ICM} \geqslant 30$

10. Classe funcional pré-operatória

11. Infarto do miocárdio prévio

12. Indice cárdiotorácico

13. Pressão diastólica final de ventrículo esquerdo pré-operatório

14. Número de ramos arteriais acometido

15. Número de enxertos realizados

16. Utilização da artéria "mamária"

17. Acometimento do tronco coronariano

18. Tipo de proteção miocárdica

19. Duração da circulação extracorpórea

20. Tempo de pinçamento aórtico

21. Baixo débito cardiaco no pós-operatório

22. Uso de balâo intraórtico

23. Acidente vascular cerebral no pós-operatório

24. Insuficiência renal no pós-operatório

25. Assistência renal no pós-operatório

26. Infecção no pós-operatório

27. Permanência hospitalar em dias
TABELA 2

MORTALIDADE HOSPITALAR MAMARIA — SAFENA

\begin{tabular}{lccc}
\hline & Com "mamária" & Com safena & \\
\hline Vivos & 45 & 45 & 90 \\
Óbitos & 2 & 8 & 10 \\
& 47 & 53 & \\
\hline
\end{tabular}

$(p<0,05)$.

\section{CASUISTICA E MÉTODOS}

Com a finalidade de se atingir o objetivo proposto, foi determinado o número de amostra, que foi constituído por 100 pacientes. Um estudo prospectivo, baseado em roteiro elaborado especificamente para este fim, foi iniciado. Neste roteiro foram incluídos parâmetros clínicos, radiológicos, hemodinâmicos, operatórios e de pós-operatórios.

Os resultados foram comparados utilizando o teste do $\mathrm{X}^{2}$ de Pearson e pela prova exata de Fisher.

\section{Técnica operatoria}

Os pacientes foram operados por meio de toracotomia mediana extrapleural, hipotermia moderada, circulação extracorpórea e proteção miocárdica realizada através da solução cardioplégica do tipo Saint Thomas ou pelo pinçamento intermitente.

As anastomoses distais foram feitas em chuleio contínuo de fio $7 \times 0$ e as próximas também em chuleio de fio $6 \times 0$.

\section{RESULTADOS}

Da casuística dos 100 pacientes, 53 pertenciam ao Grupo 1 e 47 ao Grupo 2.

No Grupo 1, a média de idade foi igual a 72,53 (70 a 83), sendo 24 pacientes do sexo feminino $(45,28 \%)$. O diabetes mellitus esteve presente em 22 (41,50\%) dos pacientes. Três deles $(15,5 \%)$ haviam sido acometidos por acidente vascular cerebral previamente à operação. Quatro pacientes $(7,54 \%)$ alegavam clinicamente serem portadores de doença pulmonar obstrutiva crônica. A hipertensão arterial pré-operatória estava presente em 42 dos pacientes deste grupo $(79,24 \%)$. O tabagismo estava presente em 24 deles $(45,28 \%)$. A angina era instável em 31 dos pacientes $(58,49 \%)$. A obesidade foi constatada em nove dos pacientes $(16,98 \%)$. 
IGLÉZIAS, J. C. R.; DALLAN, L. A. O.; LOURENÇO FILHO, D. D.; FABRI, H. A.; RAMIRES, J. F.; LUZ, P. L.; OLIVEIRA, S. A.: PILEGGI, F.; JATENE, A. D. - A utilização da artéria torácica interna aumenta a mortalidade hospitalar do paciente coronariano idoso revascularizado? Rev. Bras. Cir. Cardiovasc., 6(2): 124-127, 1991.

No periodo pré-operatório, cinco pacientes $(9,43 \%)$ encontravam-se em CF I, $15(28,30 \%)$ em CF II, 21 $(39,62 \%)$ em CF III e $12(22,64 \%)$ em CF IV.

O infarto do miocárdio pré-operatório acometeu 33 pacientes do grupo $(62,26 \%)$.

$\mathrm{O}$ índice cardiotorácico era $>0,50$ em 23 deles $(43,39 \%)$. A PDFVE, foi $\geqslant 20 \mathrm{mmHg}$ em nove pacientes $(16,98 \%)$. Quarenta pacientes $(75,47 \%)$ apresentavam cromprometimento de três ou mais ramos coronários. Treze pacientes $(24,52 \%)$ receberam até dois enxertos venosos. O tronco coronariano esquerdo estava acometido em 11 pacientes $(20,75 \%)$. A proteção miocádica foi realizada por pinçamento intermitente em 38 pacientes $(71,69 \%)$ e por cardioplegia nos 15 demais $(28,3 \%)$. A média da duração da circulação extracorpórea em minutos foi de $99,01(0-254)$ e a do tempo de pinçamento aórtico, de 52,69 (0-80).

O baixo débito cardíaco foi constatado clinicamente no período pós-operatório em 10 pacientes $(18,86 \%)$. Três deles $(5,66 \%)$ necessitaram de balão intraórtico.

O acidente vascular cerebral acometeu cinco pacientes do grupo $(9,43 \%)$. A insuficiência renal com creatinina $\geqslant 3,0 \mathrm{mg} / \mathrm{dl}$ acometeu sete $(13,20 \%)$ pacientes no periodo pós-operatório. A assistência ventilatória por período $\geqslant 24$ hs foi necessária em 13 pacientes $(24,52 \%)$. A infecção acometeu $15(28,30 \%)$ dos pacientes no período pós-operatório e a permanência hospitalar em dias foi igual a 23,9 (4-65).

O Grupo 2 era formado por 47 pacientes, sendo sete do sexo feminino $(14,98 \%)$. A média de idade foi igual a 70,48 (70-76). O diabetes mellitus acometeu 11 pacientes do grupo $(23,40 \%)$. Nenhum deles havia sido acometido por AVC previamente à operação. Três $(6,38 \%)$ pacientes eram portadores de doença pulmonar obstrutiva crônica. A hipertensão arterial foi constatada em 35 dos pacientes do grupo $(74,46 \%)$. Vinte e três pacientes $(48,93 \%)$ eram tabagistas. A angina era instável em 16 pacientes $(34,04 \%)$.

A obesidade foi constatada em 7 (14,89\%) dos pacientes do grupo. Pré-operatoriamente, nove pacientes $19,14 \%$ se encontravam em CF I, 16 (34,04\%) em CF II, $17(36,17 \%)$ em CF III e cinco $(10,63 \%)$ em CF IV. $\mathrm{O}$ infarto do miocárdio havia acometido 10 pacientes $(40,42 \%)$ do grupo, antes da operação. O índice cardiotorácico foi $\geqslant 0,50$ em 17 pacientes $(36,17 \%)$. A PDF Ve era $\geqslant 20 \mathrm{mmHg}$ no período pré-operatório em 11 pacientes $(23,40 \%)$. Trinta $(63,82 \%)$ pacientes apresentavam acometimento de três ou mais ramos coronários, sendo que $32(68,08 \%)$ receberam três ou mais enxertos. Um $(2,12 \%)$ paciente do grupo apresentava acometimento do tronco coronário esquerdo. A proteçăo miocárdica foi realizada por pinçamento intermitente em 37 pacientes $(78,72 \%)$ e por solução cardioplégica em 10 $(21,27 \%)$.

O tempo médio da circulação extracorpórea em minutos foi igual a $94,42(50-180)$ e o de pinçamento aórti$\mathrm{co}$, de 48,80 (17-93). O baixo débito cardíaco foi diagnosticado clinicamente em três pacientes $(5,66 \%)$ no período pós-operatório, sendo que nenhum necessitou de balāo intraórtico. Quatro pacientes $(8,5 \%)$ foram acometidos de acidente vascular cerebral no período pós-operatório. $A$ insuficiência renal, com creatinina $\geqslant 24$ horas, foi necessária em quatro pacientes $(8,5 \%)$.

Quatro pcientes foram acometidos de infecção no período pós-operatório $(8,5 \%)$.

A média de permanência hospitalar para o grupo foi igual a 17,12 dias (9-32).

A análise estatística revelou que houve significância estatística entre os subgrupos de pacientes idosos ( $\geqslant$ 70 anos) submetidos a revascularização do miocárdio e que a mortalidade hospitalar foi maior $8 / 53$ e 2/47 no subgrupo revascularizado utilizando-se enxertos de veia safena autógena ( $p<0,05)$.

No Grupo dos 53 pacientes, houve oito óbitos na análise de trinta dias e no Grupo dos 47 pacientes, dois haviam ido a obito.

\section{DISCUSSÃO}

Anteriormente, os pacientes com idade superior a 65 anos eram recusados para revascularização do miocárdio, com base na idade (ENNABLI \& PELLETIEN ${ }^{3}$ ).

Com a melhoria nos recursos tecnológicos, das técnicas operatórias e na proteção miocárdica, um número crescente de pacientes foi aceito para a operaçăo, e com bons resultados.

No período pós-operatório dos pacientes idosos, observa-se um número maior de complicaçōes neurológicas, pulmonares, renais e infecciosas, o que aumenta a permanência hospitalar, porém não contra-indica a operação.

Baseados em dados obtidos pelo autor, ainda não publicados, quando na identificação de fatores de risco para a revascularização do paciente coronariano idoso, foram identificados tabagismo, parâmetros de disfunção ventricular esquerda e utilizaçāo da artéria torácica interna.

Esta última constatação aparentemente discordava dos dados da literatura, os quais concluiam que a operação feita com utilização de artéria mamária interna não só é mais segura, como não está associada ao aumento 
IGLÉZIAS, J. C. R.; DALLAN, L. A. O.; LOURENÇO FILHO, D. D.; FABRI, H. A.; RAMIRES, J. F.; LUZ, P. L.; OLIVEIRA, S. A.; PILEGGI, F.; JATENE, A. D. - A utilização da artéria torácica interna aumenta a mortalidade hospitalar do paciente coronariano idoso revascularizado? Rev. Bras. Cir. Cardiovasc., 6(2): 124-127, 1991.

da morbidade ou mortalidade (COSGROVE et alii $^{2}$ ), além de permitir melhor proteção contra as manifestaçōes da doença arterial, quando comparada ao enxerto de veia safena autógena.

Baseados em informaçōes, coincidentes com aquelas de CAMERON et alii ${ }^{1}$, de que a utilização está relacionada com maior morbidade, permanência hospitalar e mortalidade, desenvolvemos o protocolo e concluímos que a mortalidade hospitalar é maior nos pacientes coronarianos idosos (idade $\geqslant 70$ anos) submetidos a revascularização do miocárdio e que receberam enxertos de veia safena autógena ( $p>0,05)$.

Atribuímos o resultado obtido ao fato de o cirurgiāo proceder à escolha do paciente que irá receber o enxerto de artéria torácica interna, aplicando-o aos pacientes em melhor estado geral e funcionando, portanto, como triador.

\section{RBCCV 44205-141}

IGLÉZIAS, J. C. R.; DALLAN, L. A. O.; LOURENÇO FILHO, D. D.; FABRI, H. A.; RAMIRES, J. F.; LUZ, P. L.; OlIVEIRA, S. A.; PILEGGI, F.; JATENE, A. D. - Does the use of the internal thoracic artery enhance the nosocomial mortality of the elderly revascularized patient? Rev. Bras. Cir. Cardiovasc., 6(2): 124-127, 1991.

ABSTRACT: At the Heart Institute, we performed a study to evaluate if the internal thoracic artery modified the nosocomial mortality of elderly coronary patients (age $\geqslant 70$ years), submitted to elective isolated revascularization of the myocardium. Clinical, hemodynamic, radiologic, operative and postoperative parameters were included in the protocol. In a prospective study one hundred consecutive patients, with complete observations were analysed. The statistical analysis was performed using Pearson's $X^{2}$ test. The results of this study showed that there was a statistically significant difference between the groups, the nosocomial mortality being higher in that in which autogenous saphenous vein grafts were used. The role of the surgeon as a screener regarding obtention of results is also discussed.

DESCRIPTORS: myocardial revascularisation, surgery.

\section{REFERÊNCIAS BIBLIOGRÁFICAS}

1 CAMERON, A. L.; KEMP, H. G.; GREen, G. E. - Bypass surgery with the internal mammary artery or saphenous vein graft: 15 years follow-up. Circulation, 74 (Supl. 3): 30-36, 1986.

2 COSGROVE, D. M.; LOOP, F. D.; LYTLE, B. W.; GOORMASTIC, M.; STEWART, R. W.; GIPP, C. C.; GOLDING, L. R. - Does mammary artery grafting increase surgical risb? Circulation, 72 (Supl. 2): 170-174, 1985.

3 ENNABLI K. \& PELLETIEN, C. - Morbidity and mortality of coronary artery surgery after the age of 70 years. Ann. Thorac. Surg., 42: 197-200, 1986.
4 GERSH, B. J.; KNONMAL, R. A.; FRY, R. L. - Coronary arteriography and coronary artery bypass surgery: morbidity and mortality in patients aged 65 years or older: a report from the coronary artery surgery study. Circulation, 67: 483-491, 1986.

5 GIBSON, C. F. \& LOOP, F. D. - Choice of internal mammary artery or saphenous vein graft: 15 years follow-up for myocardial revascularization. Cardiology, 73: 235-241, 1986.

6 MONTAGNE, N. T.; KOUCHOUKOS, N. T.; WILSON, T. A.; BENNETT, A. L.; KNOTT, H. W. LOCHRIDGE, S. K.; ERATH, H. G.; GLAYTON, O. W. - Morbidity and mortality of coronary bypass grafting in patient 70 years of age and older. Ann. Thorac. Surg., 39: 552-557, 1985. 